PROCEEDINGS OF THE

AMERICAN MATHEMATICAL SOCIETY

Volume 138, Number 11, November 2010, Pages 3891-3897

S 0002-9939(2010)10430-6

Article electronically published on June 4, 2010

\title{
EFFECTIVE NONVANISHING OF CANONICAL HECKE $L$-FUNCTIONS
}

\author{
GEORGE BOXER AND PETER DIAO
}

(Communicated by Wen-Ching Winnie Li)

\begin{abstract}
Motivated by work of Gross, Rohrlich, and more recently Kim, Masri, and Yang, we investigate the nonvanishing of central values of $L$ functions of "canonical" weight $2 k-1$ Hecke characters for $\mathbb{Q}(\sqrt{-p})$, where $3<p \equiv 3(\bmod 4)$ is prime. Using the work of Rodriguez-Villegas and Zagier, we show that there are nonvanishing central values provided that $p \geq 6.5(k-1)^{2}$ and $(-1)^{k+1}\left(\frac{2}{p}\right)=1$. Moreover, we show that the num-
\end{abstract} ber of such $\psi \in \Psi_{p, k}$ satisfies

$$
\#\left\{\psi \in \Psi_{p, k} \mid L(\psi, k) \neq 0\right\} \geq \frac{h(-p)}{\# \operatorname{Cl}(K)[2 k-1]} .
$$

\section{INTRODUCTION}

Throughout we suppose that $3<p \equiv 3(\bmod 4)$ is prime. Let $K=\mathbb{Q}(\sqrt{-p})$ be the corresponding imaginary quadratic field, and let $\mathcal{O}_{K}$ be its ring of integers. A canonical Hecke character is a Hecke character $\psi$ of $K$ of weight $2 k-1$ and conductor $\sqrt{-p} \mathcal{O}_{K}$ satisfying

$$
\psi((\alpha))=\epsilon(\alpha) \alpha^{2 k-1} \quad \text { for }\left(\alpha, \sqrt{-p} \mathcal{O}_{K}\right)=1,
$$

where $\epsilon$ is defined via the isomorphism $\mathcal{O}_{K} / \sqrt{-p} \cong \mathbb{Z} / p$ and the Dirichlet character $\left(\frac{-p}{\cdot}\right)$ on $\mathbb{Z} / p$. Since the canonical Hecke characters are determined on principal ideals, there are precisely $h(-p)$ of them, and if $\psi$ is any one of them, they are given by

$$
\Psi_{p, k}=\left\{\psi \chi: \chi \in \mathrm{Cl}(K)^{\wedge}\right\} .
$$

The $L$-function associated to a canonical Hecke character is

$$
L(\psi, s)=\sum_{0 \neq \mathfrak{a} \subset \mathcal{O}_{K}} \frac{\psi(\mathfrak{a})}{N(\mathfrak{a})^{s}} \quad \text { for } \quad \Re(s)>k+\frac{1}{2} .
$$

It has an analytic continuation to $\mathbb{C}$ and satisfies a functional equation under $s \mapsto$ $2 k-s$ with root number $(-1)^{k+1}\left(\frac{2}{p}\right)$ (see [RVZ, p. 86]).

Our main result is the following effective nonvanishing theorem for the central values $L(\psi, k)$.

Received by the editors November 3, 2009 and, in revised form, February 4, 2010.

2010 Mathematics Subject Classification. Primary 11M99.

(C)2010 American Mathematical Society Reverts to public domain 28 years from publication 
Theorem 1. If $k$ is a positive integer and $3<p \equiv 3(\bmod 4)$ is a prime for which $p \geq 6.5(k-1)^{2}$ and $(-1)^{k+1}\left(\frac{2}{p}\right)=1$, then

$$
\frac{1}{h(-p)} \sum_{\psi \in \Psi_{p, k}} L(\psi, k) \geq \frac{\pi^{k} p^{k / 2-3 / 4}}{18^{k-1}(k-1) !} e^{-\pi \sqrt{p} / 4} .
$$

In particular, there exists at least one $\psi \in \Psi_{p, k}$ such that $L(\psi, k)>0$.

Remark 2. One can obtain this result for general fundamental discriminants of imaginary quadratic fields $-3>d \equiv 1(\bmod 4)$ using Rodriguez-Villegas and Zagier formulae holding in that case (see [RV, p. 436] or [RVY, p. 561]). One obtains the same lower bound in the same range.

These central values first arose in connection with work of Gross $\mathrm{Gr}$ and Rohrlich [R, R2 on ranks of elliptic $\mathbb{Q}$-curves. More recently, Kim, Masri, and Yang KMY have shown under some technical conditions that the nonvanishing of these central values implies that certain Selmer groups associated to the characters $\psi$ are finite. The nonvanishing of the central values $L(\psi, k)$ under the assumption that $(2 k-1, h(-p))=1$ has been studied extensively by Rohrlich, Rodriguez-Villegas, Zagier, Yang, and many others (see for example [R, R2, MR, RVZ, MiY, Y, LX]).

When $(2 k-1, h(-p))>1, \Psi_{p, k}$ breaks up into multiple Galois orbits. We recall that the Galois group $G=\operatorname{Gal}(\overline{\mathbb{Q}} / K)$ acts on $\Psi_{p, k}$ by

$$
\psi^{\sigma}(\mathfrak{a})=\psi(\mathfrak{a})^{\sigma} \quad \text { for } \quad \sigma \in G,
$$

where we have fixed an embedding $K \subset \overline{\mathbb{Q}}$. By [3] and [Ma2, Prop. 1.1], for any $\psi \in \Psi_{p, k}$,

$$
\#\left\{\psi^{\sigma} \mid \sigma \in G\right\}=\frac{h(-p)}{\# \mathrm{Cl}(K)[2 k-1]},
$$

where $\mathrm{Cl}(K)[2 k-1]$ is the $(2 k-1)$-torsion subgroup of $\mathrm{Cl}(K)$. By an important theorem of Shimura [Sh, p. 212], for $\sigma \in G$ and $\psi \in \Psi_{p, k}$,

$$
L\left(\psi^{\sigma}, k\right) \neq 0 \quad \Longleftrightarrow \quad L(\psi, k) \neq 0 .
$$

The following corollary is now an immediate consequence of Theorem 1 .

Corollary 3. Under the same assumptions on $p$ and $k$ as in Theorem 1 we have

$$
\#\left\{\psi \in \Psi_{p, k} \mid L(\psi, k) \neq 0\right\} \geq \frac{h(-p)}{\# \operatorname{Cl}(K)[2 k-1]} .
$$

Note that Siegel's lower bound $h(-p) \gg p^{1 / 2-\epsilon}$ and the widely believed upper bound \# $\mathrm{Cl}(K)[2 k-1] \ll p^{\epsilon}$ would imply

$$
\#\left\{\psi \in \Psi_{p, k} \mid L(\psi, k) \neq 0\right\} \gg p^{1 / 2-\epsilon} .
$$

In [Ma, Masri proves nonvanishing theorems for canonical Hecke $L$-functions which are valid even when $(2 k-1, h(-p))>1$. He uses a formula of RodriguezVillegas and Zagier [RVZ] for $L(\psi, k)$ as well as equidistribution of Heegner points to obtain an asymptotic formula for the first moment

$$
\frac{1}{h(-p)} \sum_{\psi \in \Psi_{p, k}}|L(\psi, k)|
$$


as $p$ tends to infinity. From here he uses a subconvexity bound of Duke, Friedlander, and Iwaniec [DFI] to obtain a nonvanishing theorem of the form

$$
\#\left\{\psi \in \Psi_{p, k} \mid L(\psi, k) \neq 0\right\} \gg p^{\delta}
$$

for some $\delta>0$ and all $p$ sufficiently large. However, this result is ineffective due to an application of Siegel's theorem; thus one cannot say how large $p$ must be taken to guarantee the existence of a nonvanishing central value.

The Rodriguez-Villegas and Zagier formula relates $L(\psi, k)$ to a sum of a fixed theta series evaluated at CM points. Our main observation in this paper is that we can quantify the nonvanishing problem by studying the position of the CM point corresponding to the trivial ideal class in the cusp. This idea of quantification in the cusp also appears in work of Michel and Venkatesh [MV] on nonvanishing of Rankin-Selberg $L$-functions.

\section{The formula of Rodriguez-Villegas and Zagier}

In this section we recall the Rodriguez-Villegas and Zagier formula for the central values of canonical Hecke $L$-functions. In the notation of [RVZ], we first define the real-analytic theta series $\theta_{l+1 / 2}$ for $l \geq 0$ by

$$
\theta_{l+1 / 2}(z)=\frac{1}{(2 \pi y)^{l / 2}} \sum_{\substack{n \geq 1 \\ n \text { odd }}}\left(\frac{-4}{n}\right)^{l} H_{l}(n \sqrt{\pi y / 2}) e^{\pi i n^{2} z / 4},
$$

where $H_{l}$ is the Hermite polynomial defined by

$$
H_{l}(z)=\sum_{0 \leq j \leq l / 2} \frac{l !}{j !(l-2 j) !}(-1)^{j}(2 z)^{l-2 j} .
$$

Next, we define Heegner points suitable for evaluating modular forms of level a power of 2. Given a primitive ideal $\mathfrak{a}=\left[a, \frac{b+\sqrt{-p}}{2}\right]$ prime to $p$, we are free to choose $b$ congruent to 1 modulo 16 and then we define

$$
z_{\mathfrak{a}}^{(2)}=\frac{b+\sqrt{-p}}{2 a} \in \mathbb{H} .
$$

With notation as above we recall the following theorem of Rodriguez-Villegas and Zagier.

Theorem $4(\mathrm{RVZ})$. Let $3<p \equiv 3(\bmod 4)$ be a prime and let $\psi$ be a canonical Hecke character of weight $2 k-1$ associated to $K$ with root number 1 . Then one has the following identity:

$$
L(\psi, k)=\frac{\pi^{k} p^{k / 2-3 / 4}}{2^{k-3}(k-1) !}\left|\sum_{[\mathfrak{a}] \in \mathrm{Cl}(K)}\left(\frac{-4}{N(\mathfrak{a})}\right)^{\delta} \overline{\psi(\mathfrak{a})}^{-1} \theta_{k-1 / 2}\left(z_{\mathfrak{a}^{2}}^{(2)}\right)\right|^{2},
$$

where $\delta$ is defined by $\left(\frac{2}{p}\right)=(-1)^{\delta}$. 


\section{Bounds For HeRmite POLYNOMIALS}

In this section we establish some facts concerning Hermite polynomials that will be used in the proof of Theorem 1. First we need the following bound on the largest zero of the Hermite polynomial $H_{l}$.

Proposition 5 ([K, p. 36]). Let $l \geq 1$. Then $H_{l}(x)$ has no zeros for $x \geq \sqrt{2 l-2}$. In particular, $H_{l}(x)$ is positive in that range.

Using this we can obtain upper and lower bounds for $H_{l}(x)$.

Lemma 6. For $x>\sqrt{2 l}$ we have

$$
(2 x / 3)^{l} \leq H_{l}(x) \leq(2 x)^{l} .
$$

Proof. First we prove the upper bound. It is clear for $l=0,1$ from the expressions $H_{0}(x)=1, H_{1}(x)=2 x$. For $l \geq 2$ we proceed inductively using the standard recurrence

$$
H_{l}(x)=2 x H_{l-1}(x)-2 l H_{l-2}(x) .
$$

For $x>\sqrt{2 l}$, Proposition 5 implies $H_{l-2}(x) \geq 0$ and so

$$
H_{l}(x) \leq 2 x H_{l-1}(x) \leq(2 x)^{l}
$$

by induction. Similarly, the lower bound is immediately verified for $l=0,1$. For $l \geq 2$ we denote the largest real root of $H_{l}(x)$ by $r_{l}$. We use the standard fact that

$$
H_{l}^{\prime}(x)=2 l H_{l-1}(x) \text {. }
$$

For $y>\sqrt{2 l}$ we can write

$$
H_{l}(y)=\int_{r_{l}}^{y} H_{l}^{\prime}(x) d x=\int_{r_{l}}^{y} 2 l H_{l-1}(x) d x \geq \int_{\sqrt{2 l-2}}^{y} 2 l H_{l-1}(x) d x,
$$

where the last step is justified by Proposition [5 and $r_{l}>r_{l-1}$. Inductively using the fact that $H_{l-1}(x) \geq(2 x / 3)^{l-1}$ in the range $x>\sqrt{2 l-2}$, we find that

$$
H_{l}(y) \geq \int_{\sqrt{2 l-2}}^{y} 2 l\left(\frac{2 x}{3}\right)^{l-1} d x=3\left(\frac{2}{3}\right)^{l}\left[y^{l}-(2 l-2)^{l / 2}\right] .
$$

It remains to show that for $y>\sqrt{2 l}$,

$$
3\left(\frac{2}{3}\right)^{l}\left[y^{l}-(2 l-2)^{l / 2}\right] \geq\left(\frac{2}{3}\right)^{l} y^{l},
$$

which is equivalent to

$$
2 y^{l} \geq 3(2 l-2)^{l / 2} .
$$

For $y>\sqrt{2 l}$ this follows from

$$
\frac{2}{3}>\frac{1}{\sqrt{e}}>\left[1-\frac{1}{l}\right]^{l / 2} .
$$

Remark 7. Since $H_{l}(x) \sim(2 x)^{l}$ as $x$ tends to infinity, the lower bound in the above lemma is far from optimal for $x$ large. However, we are primarily interested in the lower bound in the region where $x$ is not much larger than the largest zero of $H_{l}$. 


\section{Proof of Theorem 1}

We first use the Rodriguez-Villegas and Zagier formula to obtain an exact formula for the first moment. By Theorem [4 we have that

$$
\frac{1}{h(-p)} \sum_{\psi \in \Psi_{p, k}} L(\psi, k)=\frac{C_{k}}{h(-p)} \sum_{\psi \in \Psi_{p, k}}\left|\sum_{[\mathfrak{a}] \in \mathrm{Cl}(K)}\left(\frac{-4}{N(\mathfrak{a})}\right)^{\delta} \overline{\psi(\mathfrak{a})}-1 \theta_{k-1 / 2}\left(z_{\mathfrak{a}^{2}}^{(2)}\right)\right|^{2}
$$

where we denote

$$
C_{k}=\frac{\pi^{k} p^{k / 2-3 / 4}}{2^{k-3}(k-1) !}
$$

Recall that

$$
\Psi_{p, k}=\left\{\psi_{0} \chi \mid \chi \in \mathrm{Cl}(K)^{\wedge}\right\}
$$

for a fixed $\psi_{0} \in \Psi_{p, k}$. Squaring out the summand, interchanging orders of summation, and using the orthogonality relations yield

$$
\begin{aligned}
& \frac{1}{h(-p)} \sum_{\psi \in \Psi_{p, k}} L(\psi, k) \\
& =C_{k} \sum_{[\mathfrak{a}],[\mathfrak{b}] \in \mathrm{Cl}(K)} \overline{\psi_{0}(\mathfrak{a} \overline{\mathfrak{b}})}{ }^{-1} \theta_{k-1 / 2}\left(z_{\mathfrak{a}^{2}}^{(2)} \overline{\theta_{k-1 / 2}\left(z_{\mathfrak{b}^{2}}^{(2)}\right)}\left(\frac{-4}{N(\mathfrak{a} \mathfrak{b})}\right)^{\delta} \frac{1}{h(-p)} \sum_{\chi \in \mathrm{Cl}(K)^{\wedge}} \chi(\overline{\mathfrak{a} \mathfrak{b})}\right. \\
& =C_{k} \sum_{[\mathfrak{a}] \in \mathrm{Cl}(K)} \psi_{0}(\mathfrak{a} \overline{\mathfrak{a}})^{-1}\left|\theta_{k-1 / 2}\left(z_{\mathfrak{a}^{2}}^{(2)}\right)\right|^{2} .
\end{aligned}
$$

Using the fact that

$$
\psi_{0}(\mathfrak{a} \overline{\mathfrak{a}})=\psi_{0}(N(\mathfrak{a}))=N(\mathfrak{a})^{2 k-1},
$$

we obtain the following exact formula for the first moment:

$$
\frac{1}{h(-p)} \sum_{\psi \in \Psi_{p, k}} L(\psi, k)=\frac{\pi^{k} p^{k / 2-3 / 4}}{2^{k-3}(k-1) !} \sum_{[\mathfrak{a}] \in \mathrm{Cl}(K)} \frac{1}{N(\mathfrak{a})^{2 k-1}}\left|\theta_{k-1 / 2}\left(z_{\mathfrak{a}^{2}}^{(2)}\right)\right|^{2} .
$$

Using positivity, we obtain the lower bound

$$
\frac{1}{h(-p)} \sum_{\psi \in \Psi_{p, k}} L(\psi, k) \geq \frac{\pi^{k} p^{k / 2-3 / 4}}{2^{k-3}(k-1) !}\left|\theta_{k-1 / 2}\left(z_{\mathcal{O}_{K}}^{(2)}\right)\right|^{2}
$$

where

$$
z_{\mathcal{O}_{K}}^{(2)}=\frac{1+\sqrt{-p}}{2}
$$

is the Heegner point corresponding to the trivial ideal class. The Fourier expansion of $\theta_{k-1 / 2}$ at the cusp at infinity evaluated at the trivial Heegner point is

$$
\begin{aligned}
\theta_{k-1 / 2} & \left(\frac{1+\sqrt{-p}}{2}\right) \\
& =\frac{1}{(\pi \sqrt{p})^{\frac{k-1}{2}}} \sum_{n \geq 1}\left(\frac{-4}{n}\right)^{k-1} H_{k-1}\left(\frac{n \pi^{1 / 2} p^{1 / 4}}{2}\right) e^{\pi i n^{2} x / 8} e^{-\pi n^{2} \sqrt{p} / 8} .
\end{aligned}
$$

For convenience, let $a_{n}$ denote the $n$th term in this sum (note that $a_{n}=0$ for $n$ even). The strategy is to bound the first term below in magnitude while comparing 
the rest of the terms to a geometric series by bounding the magnitude of the ratios of consecutive terms. For

$$
\frac{\pi^{1 / 2} p^{1 / 4}}{2} \geq \sqrt{2 k-2}
$$

we can use the estimates in Lemma 6 to obtain, for any integer $m \geq 0$,

$$
\begin{aligned}
\left|\frac{a_{2 m+3}}{a_{2 m+1}}\right| & =\left|\frac{H_{k-1}\left(\frac{(2 m+3) \pi^{1 / 2} p^{1 / 4}}{2}\right) e^{-\pi(2 m+3)^{2} \sqrt{p} / 8}}{H_{k-1}\left(\frac{(2 m+1) \pi^{1 / 2} p^{1 / 4}}{2}\right) e^{-\pi(2 m+1)^{2} \sqrt{p} / 8}}\right| \\
& \leq\left(\frac{2 m+3}{2 m+1}\right)^{2 k-2} 3^{2 k-2} e^{-\pi(m+1) \sqrt{p}} \\
& \leq 81^{k-1} e^{-\pi \sqrt{p}}
\end{aligned}
$$

The above inequality holds for (5), which is true for $p \geq 6.5(k-1)^{2}$. Since $p \geq 7$, we uniformly obtain

$$
81^{k-1} e^{-\pi \sqrt{p}} \leq \frac{1}{3}
$$

Thus under these assumptions we have that

$$
\left|a_{2 m+1}\right| \leq \frac{\left|a_{1}\right|}{3^{m}}
$$

Now we have

$$
\begin{aligned}
\left|\theta_{k-1 / 2}\left(z_{1}^{(2)}\right)\right| & \geq \frac{1}{(\pi \sqrt{p})^{\frac{k-1}{2}}}\left(\left|a_{1}\right|-\sum_{m=1}^{\infty}\left|a_{2 m+1}\right|\right) \\
& \geq \frac{1}{(\pi \sqrt{p})^{\frac{k-1}{2}}}\left|H_{k-1}\left(\frac{\pi^{1 / 2} p^{1 / 4}}{2}\right) e^{-\pi \sqrt{p} / 8}\right|\left(1-\frac{1}{3}-\frac{1}{3^{2}}-\frac{1}{3^{3}} \ldots\right) \\
& =\frac{1}{(\pi \sqrt{p})^{\frac{k-1}{2}}} \frac{1}{2}\left|H_{k-1}\left(\frac{\pi^{1 / 2} p^{1 / 4}}{2}\right) e^{-\pi \sqrt{p} / 8}\right| \\
& \geq \frac{1}{3^{k-1}} \frac{1}{2} e^{-\pi \sqrt{p} / 8}>0
\end{aligned}
$$

where we used the lower bound of Lemma 6 in the last step. For $p \geq 6.5(k-1)^{2}$, substituting this lower bound into (4) yields

$$
\frac{1}{h(-p)} \sum_{\psi \in \Psi_{p, k}} L(\psi, k) \geq \frac{\pi^{k} p^{k / 2-3 / 4}}{18^{k-1}(k-1) !} e^{-\pi \sqrt{p} / 4} .
$$

\section{ACKNOWLEDGEMENTS}

We are grateful to Riad Masri and Ken Ono for their guidance and support throughout this project. We also thank the anonymous referee for pointing out that our argument generalizes to general fundamental discriminant. 


\section{REFERENCES}

[DFI] Duke, W., J. Friedlander, and H. Iwaniec, Bounds for Automorphic L-functions. II. Inventiones Mathematicae 115 (1994), 219-239. MR1258904 (95a:11044)

[Gr] B. Gross, Arithmetic on elliptic curves with complex multiplication. With an appendix by B. Mazur. Lecture Notes in Mathematics, 776. Springer, Berlin, 1980. iii+95 pp. MR563921 (81f:10041)

[KMY] B. D. Kim, R. Masri, and Tonghai Yang, Nonvanishing of Hecke L-functions and the Bloch-Kato conjecture, preprint.

[K] Krasikov, Ilia, Nonnegative Quadratic Forms and Bounds on Orthogonal Polynomials. Journal of Approximation Theory 111 (2001), 31-49. MR1840019 (2002j:42034)

[LX] C. Liu and L. Xu, The vanishing order of certain Hecke L-functions of imaginary quadratic fields. J. Number Theory 108 (2004), 76-89. MR.2078658 (2005g:11221)

[Ma] R. Masri, Asymptotics for sums of central values of canonical Hecke L-series. Int. Math. Res. Not. IMRN 2007, no. 19, Art. ID rnm065, 27 pp. MR2359540(2009b:11090)

[Ma2] R. Masri, Quantitative nonvanishing of $L$-series associated to canonical Hecke characters. Int. Math. Res. Not. IMRN 2007, no. 19, Art. ID rnm070, 16 pp. MR2359543 (2008j:11170)

[MV] P. Michel and A. Venkatesh, Heegner points and nonvanishing of Rankin-Selberg Lfunctions. Proceedings of the Gauss-Dirichlet conference, Clay Math. Proc., 7, Amer. Math. Soc., Providence, RI, 2007. MR2362200 (2009a:11116)

[MiY] S. D. Miller and T. H. Yang, Nonvanishing of the central derivative of canonical Hecke L-functions. Math. Res. Lett. 7 (2000), 263-277. MR.1764321 (2001i:11058)

[MR] H. Montgomery and D. Rohrlich, On the L-functions of canonical Hecke characters of imaginary quadratic fields. II. Duke Math. J. 49 (1982), 937-942. MR683009 (84e:12014)

[RV] F. Rodriguez-Villegas, Square root formulas for central values of Hecke L-series II. Duke Math. J. 72 (1993), 431-440. MR1248679 (95d:11156)

[RVY] F. Rodriguez-Villegas and T. Yang, Central values of Hecke L-functions of CM number fields, Duke Math. J. 98 (1999), 541-564. MR1695801 (2000j:11074)

[RVZ] F. Rodriguez-Villegas and D. Zagier, Square roots of central values of Hecke L-series. Advances in number theory (Kingston, ON, 1991), 81-99, Oxford Sci. Publ., Oxford Univ. Press, New York, 1993. MR 1368412 (96j:11069)

[R] D. Rohrlich, The nonvanishing of certain Hecke L-functions at the center of the critical strip. Duke Math. J. 47 (1980), 223-232. MR.563377(81k:12017)

[R2] D. Rohrlich, On the L-functions of canonical Hecke characters of imaginary quadratic fields. Duke Math. J. 47 (1980), 547-557. MR587165 (81m:12020)

[R3] D. Rohrlich, Galois conjugacy of unramified twists of Hecke characters. Duke Math. J. 47 (1980), 695-703. MR.587174 (82a:12009)

[Sh] G. Shimura, On periods of modular forms. Math. Ann. 229 (1977), 211-221. MR0463119 $(57: 3080)$

[Y] T. H. Yang, Nonvanishing of central Hecke L-values and rank of certain elliptic curves. Compos. Math. 117 (1999), 337-359. MR1702416 (2001a:11093)

Frist Center, Princeton University, Mailbox 2704, Princeton, New Jersey 08544

Frist Center, Princeton University, Mailbox 2868, Princeton, New Jersey 08544 